\title{
Constraints from the CHARM experiment on heavy neutral leptons with tau mixing
}

\author{
Iryna Boiarska, ${ }^{1, *}$ Alexey Boyarsky, ${ }^{2, \dagger}$ Oleksii Mikulenko®, ${ }^{2, \ddagger}$ and Maksym Ovchynnikov $\circledast^{2, \S}$ \\ ${ }^{1}$ Discovery Center, Niels Bohr Institute, Copenhagen University, \\ Blegdamsvej 17, DK-2100 Copenhagen, Denmark \\ ${ }^{2}$ Instituut-Lorentz for Theoretical Physics, Universiteit Leiden, \\ Niels Bohrweg 2, 2333 CA Leiden, Netherlands
}

(Received 10 August 2021; accepted 6 October 2021; published 23 November 2021)

\begin{abstract}
We reanalyze the results of the searches for Heavy Neutral Leptons (HNLs) by the CHARM experiment. We study HNL decay channel $N \rightarrow e^{+} e^{-} \nu / \mu^{+} \mu^{-} \nu$ and show that, in addition to the constraints on the HNL's mixings with $\nu_{e}$ or $\nu_{\mu}$, the same data also implies limits on the HNLs that mix only with $\nu_{\tau}$ and have masses in the range $290 \mathrm{MeV}<m_{N} \lesssim 1.6 \mathrm{GeV}$ : the region in the parameter space that was considered in the literature as a target for HNLs searches.
\end{abstract}

DOI: 10.1103/PhysRevD.104.095019

\section{INTRODUCTION AND SUMMARY}

The Standard Model (SM) of particle physics, despite its unprecedented success, has proven to be incomplete, encouraging searches for new particles. Heavy Neutral Leptons, or HNLs, are a well-motivated extension of the SM that are capable of simultaneously explaining several long-standing problems: neutrino oscillations [1], dark matter [2-4], and baryon asymmetry of the Universe [58]. From the phenomenological point of view, such particles participate in weak interactions and behave as heavy neutrinos with interaction strength suppressed by the mixing angles $U_{\alpha}$, as compared to ordinary neutrinos $\nu_{\alpha}$ (see, e.g., [9]). HNLs with the masses in the $\mathrm{MeV}-\mathrm{GeV}$ range are searched for at the accelerators (see, e.g., $[10,11])$, and may be constrained from cosmological observations as well [12-14].

In the minimal models where two or three HNLs explain neutrino flavor oscillation data via a seesaw mechanism, each of the HNLs should have comparable mixings with all active neutrino flavors (see, e.g., [15-17] and references therein for discussion). Nevertheless, to obtain the accelerator bounds, it is convenient to consider HNLs that mix with only one flavor of active neutrinos, namely $\nu_{\alpha}$ (below,

\footnotetext{
*boiarska@nbi.ku.dk

†boyarsky@lorentz.leidenuniv.nl

*mikulenko@lorentz.leidenuniv.nl

§ovchynnikov@lorentz.leidenuniv.nl
}

Published by the American Physical Society under the terms of the Creative Commons Attribution 4.0 International license. Further distribution of this work must maintain attribution to the author(s) and the published article's title, journal citation, and DOI. Funded by SCOAP. we denote such HNLs as $N_{\alpha}$ ). Limits on $U_{\alpha}^{2}$ derived for such simplified "pure mixing model" are conservative, as the presence of additional mixing angles $U_{\beta \neq \alpha}^{2} \neq 0$ for the same particle would only increase the number of expected events in a given experiment at the lower bound of sensitivity.

The current bounds on $U_{e}^{2}$ and $U_{\tau}^{2}$ for HNLs with pure mixing are shown in Fig. 2 as reported in [11]. In the $\mathrm{GeV}$ mass range, the constraints on the mixing angle $U_{\tau}^{2}$ are orders of magnitude weaker as compared to the constraints on $U_{e / \mu}^{2}$ (constraints for the $\mu$ mixing are similar to the ones for the $e$ mixing). Namely, for the $e / \mu$ mixing, the large values of the couplings for HNLs with masses $m_{K} \lesssim m_{N} \lesssim$ $m_{D} \simeq 2 \mathrm{GeV}$ are excluded by the CHARM experiment $[18,19]$, while for the $\tau$ mixing CHARM constraints on $U_{\tau}$ are reported in the literature only for masses $m_{N}<290 \mathrm{MeV}$. The reason is the following: The original analysis $[18,19]$ is based on negative results for searches for decays of feebly interacting particles into one of the possible dilepton pair- $-\mu e, \mu \mu, \mu e$. For HNLs, they consider only decays mediated through the charged current (CC) interaction [see Fig. 1, diagram (a)] that give rise to leptonic decays

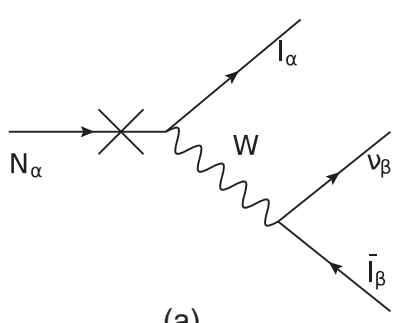

(a)

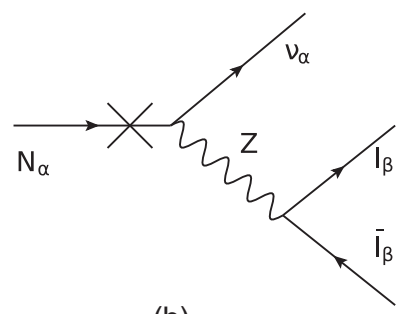

(b)
FIG. 1. Diagrams of leptonic decays of an HNL that mixes purely with $\nu_{\alpha}$ via the charged (a) and the neutral current (b). 

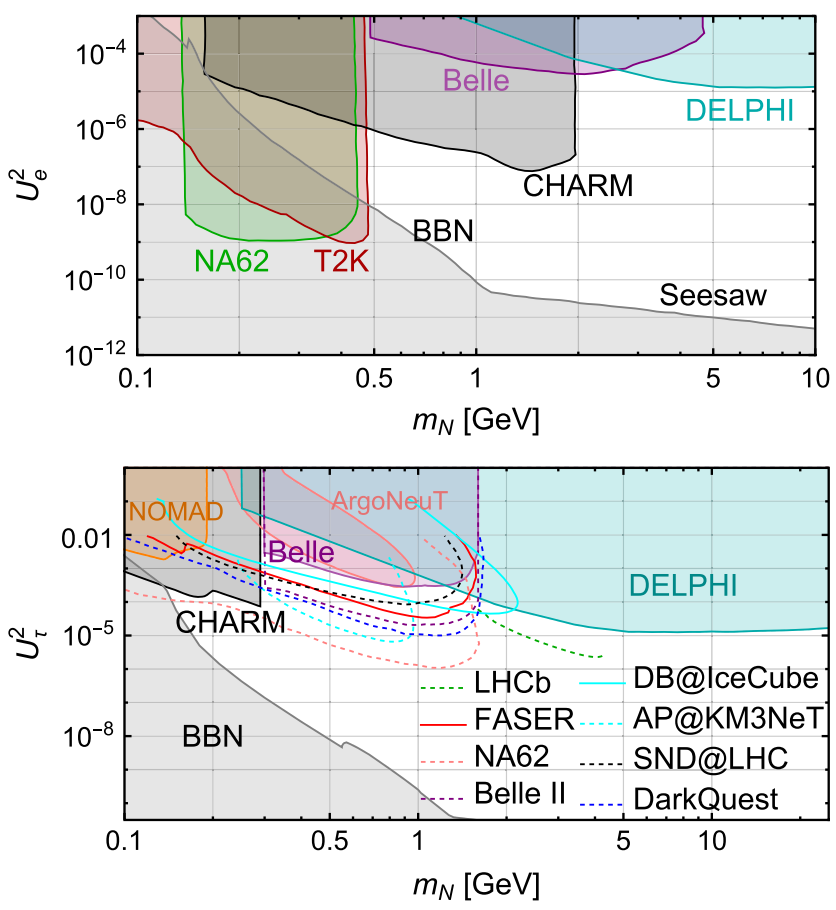

FIG. 2. The parameter space of HNLs with the pure $e$ (the top panel) and $\tau$ (the bottom panel) mixing. The current bounds are from CHARM [19,20], NA62 [21], T2K [22], Belle [23,24], DELPHI [25], NOMAD [26], ArgoNeuT [27]; see also [11] for a review and the references therein. For the pure $\tau$ mixing, we do not show the constraints imposed by the T2K experiment (unlike how it is done in [11]), since they are reported for nonzero couplings $U_{e / \mu}$ which dominate the production (The T2K experiment [22] is based on a search for decays of HNLs produced from kaons $K \rightarrow l_{\alpha} N$. Such decays can occur only through the $e / \mu$ mixings due to the small mass of kaon $m_{K}=493 \mathrm{MeV}$.). Constraints from the CHARM experiment are taken from the literature, while our reanalysis for them is shown in Fig. 6. The light gray domain corresponds to couplings that are either excluded by BBN $[12,14]$ or too small to provide active neutrino masses. For the pure $\tau$ mixing, we also show sensitivities of the next generation Intensity frontier experiments (see text for details). In cyan, we show HNL parameter space that may be probed by neutrino observatories: the solid line shows the sensitivity of IceCube to the "double bang" signature from [28], while the dashed line corresponds to the sensitivity of KM3NeT to decays of HNLs produced in the atmosphere; see text and Appendix B for details.

$$
N_{\alpha} \rightarrow l_{\alpha} \bar{l}_{\beta} \nu_{\beta}, \quad \beta=e, \mu, \tau .
$$

If only $\mathrm{CC}$ interactions are taken into account, the search is suitable to constrain the mixing of HNLs with $\nu_{e}$ and $\nu_{\mu}$. To search for CC mediated decays via the $\tau$ mixing (which necessarily include a $\tau$ lepton), the HNL mass should be $m_{N}>m_{\tau} \simeq m_{D}$ in this model. Such HNLs are mainly produced in decays of heavy $B$ mesons, the number of which at CHARM is insufficient to provide enough events for the couplings that are not excluded (see Fig. 2).
Therefore, HNLs that mix only with $\nu_{\tau}$ cannot be constrained by CHARM data using only the decays via $\mathrm{CC}$.

In order to constrain the $\tau$ mixing angles of the light HNLs $m_{N}<m_{\tau}$, one should include the interactions via the neutral current (NC) into the analysis; see Fig. 1 [diagram (b)]. In this case, the dileptonic decays are

$$
N_{\alpha} \rightarrow \nu_{\alpha} l_{\beta} \bar{l}_{\beta}
$$

and do not require the creation of a $\tau$ lepton for the pure $\tau$ mixing.

The works [16,20,29] have reanalyzed the CHARM constraints on HNLs by including also the neutral current processes. However, their analysis was insufficient to put the bounds on the pure $\tau$ mixing in $\mathrm{GeV}$ mass range. Namely, the work [20] (the results of which are used in [11]) has limited the study of the mass range by $m_{N}<290 \mathrm{MeV}$, while [16,29] considered the decays of HNLs via neutral currents but did not include the production of HNLs from $\tau$ lepton [the diagrams (c) and (d) in Fig. 4]. As a result, these works did not report any CHARM limits on the pure $\tau$ mixing.

To conclude, in the GeV mass range of HNLs, there is a gap in the parameter space probed by past experiments, possibly only due to the lack of the analysis (see Fig. 2). To close this gap, the searches for HNLs that mix mainly with $\tau$ neutrinos were considered among scientific goals for several experiments: displaced decays at FASER [10,30], Belle II [24], SND@LHC [31], DarkQuest [32], and NA62 in the dump mode [10]; prompt decays at $\mathrm{LHCb}[33,34]$; and double bang signature at IceCube, SuperKamiokande, DUNE, and HyperKamiokande [28,35].

The planned neutrino observatory KM3NeT [36] working as an atmospheric beam dump may have sensitivity to such HNLs as well. Namely, HNLs may be produced in numerous collisions of cosmic protons with atmospheric particles, then reach the detector volume located deeply underwater in the Mediterranean Sea, and further decay into a dimuon pair inside. Such combination of decay products may be in principle distinguished from the SM events due to neutrino scatterings and penetrating atmospheric muons. We discuss this signature in more detail and estimate the sensitivity of KM3NeT to HNL produced in the atmosphere in Appendix B, and make the conclusions in Sec. IV. The resulting sensitivity is shown in Fig. 2.

Yet, the main result of this paper is to remove the abovementioned limitations of the analysis for HNLs interacting only with $\tau$ neutrinos existing in the literature, and demonstrate that the results of the CHARM experiment do imply the constraints on the $\tau$-mixing of HNLs with the masses in the range $290 \mathrm{MeV}<m_{N}<1.6 \mathrm{GeV}$ that are two orders of magnitude stronger than previously reported in the literature. Our results are shown in Fig. 6.

The CHARM bounds reanalysis presented in this paper may by similarly applied for the reanalysis of bounds 
coming from the NOMAD experiment [26]. However, due to the smaller intensity of the proton beam at NOMAD and simultaneously similar geometric acceptance of the decay volume, the bounds imposed by NOMAD are subdominant, and we therefore do not make the reanalysis in this work.

\section{CHARM EXPERIMENT}

The CHARM experiment $[18,19]$ was a proton beam dump operating at the $400 \mathrm{GeV}$ CERN SPS. The total number of exposed protons was split into $1.7 \times 10^{18}$ protons on a solid copper target and $0.7 \times 10^{18}$ on a laminated copper target with the $1 / 3$ effective density. Searches for decays of HNLs were performed in the $l_{\text {fid }}=35 \mathrm{~m}$ long decay region (see Fig. 3) defined by the two scintillator planes $\mathrm{SC} 1$ and $\mathrm{SC} 2$, located at the distance $l_{\min }=480 \mathrm{~m}$ from the copper target. The decay detector covered the $3.9 \times 10^{-5}$ sr solid angle and had the transverse dimensions $3 \times 3 \mathrm{~m}^{2}$, with the center displaced by $5 \mathrm{~m}$ from the axis. The fine-grain calorimeter at CHARM was aimed to detect inelastic scattering of electrons and muons produced in hypothetical decays of HNLs [37]. The sets of tube planes P1-P5 [38] were installed to improve the reconstruction of the decay vertex and the angular resolution.

\section{PHENOMENOLOGY OF HNLS AT CHARM}

\section{A. Production}

At the SPS energy of $400 \mathrm{GeV}$, HNLs with mass at the $\mathrm{GeV}$ scale may be produced directly either in the protontarget collisions, or in the decays of secondary particles: $B$, $D$ mesons and $\tau$ leptons. The direct HNL production competes with strong interaction processes, while the production from secondary particles-with weak interactions. As a result, the latter process is dominant even taking into account small production probability of mesons [9], and the former may be completely neglected. However, similarly to the other experiment operating at SPS, NA62 in the dump mode, the CHARM experiment has no sensitivity to the HNLs produced from $B$ mesons, implying the lower bound on the probed mass $m_{N} \lesssim m_{D_{s}} \simeq 2 \mathrm{GeV}$.

Let us define the HNL that mixes only with $\nu_{\alpha}$ by $N_{\alpha}$. Neglecting the direct production channels, the total number of $N_{\alpha}$ produced at CHARM is given by:

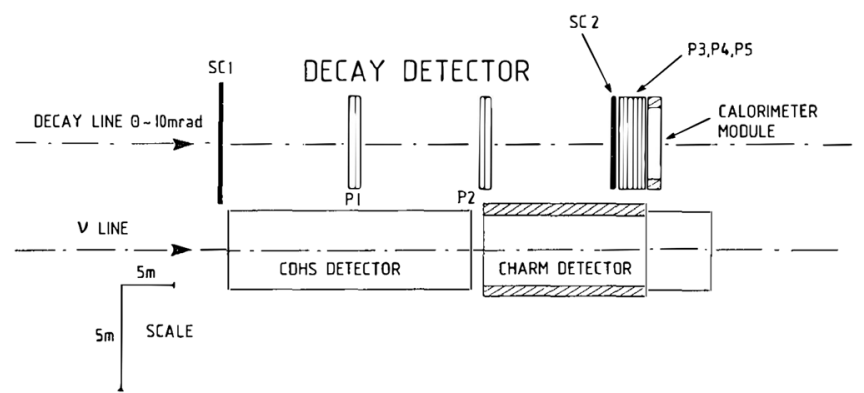

FIG. 3. The layout of the CHARM facility, adopted from [19].

$$
\begin{aligned}
\mathcal{N}_{\text {prod }}^{(\alpha)}= & 2 \mathcal{N}_{c \bar{c}} \cdot\left[\sum_{D_{i}} f_{c \rightarrow D_{i}} \operatorname{Br}\left(D_{i} \rightarrow N_{\alpha} X\right)\right. \\
& \left.+f_{c \rightarrow D_{s}} \cdot \operatorname{Br}\left(D_{s} \rightarrow \tau \bar{\nu}_{\tau}\right) \cdot \operatorname{Br}\left(\tau \rightarrow N_{\alpha} X\right)\right],
\end{aligned}
$$

with $\mathcal{N}_{c \bar{c}}$ being the total number of quark-antiquark $c \bar{c}$ pairs produced at CHARM, $D_{i}=D^{ \pm}, D^{0}, D_{s}$, and $f_{c \rightarrow D_{i}}$, the corresponding quark fragmentation fractions at SPS. The first term in the brackets describes the production from decays of $D$ mesons [diagrams (a), (b) in Fig. 4] and the second-from $\tau$ leptons in the $D_{s} \rightarrow \tau \rightarrow N$ decay chain [diagrams (c), (d) in Fig. 4]. $\operatorname{Br}\left(D_{i} \rightarrow N_{\alpha} X\right), \operatorname{Br}\left(\tau \rightarrow N_{\alpha} X\right)$ are the branching ratios. The second term includes a small factor $f_{c \rightarrow D_{s}} \cdot \operatorname{Br}\left(D_{s} \rightarrow \tau \bar{\nu}_{\tau}\right) \simeq 5 \times 10^{-3}$; for the given HNL mass, it is suppressed as compared to the first term as soon as the production from $D$ is allowed.

The original analysis of the CHARM collaboration $[18,19]$ considered the mixing $\alpha=e, \mu$, for which decays from $D$ mesons are possible for any mass in the range $m_{N}<m_{D_{s}}-m_{l_{\alpha}} \approx 1.9 \mathrm{GeV}$, and the production from $\tau$ decays may be completely neglected, according to the discussion above. For the $\tau$ mixing, however, the kinematic threshold of the production from $D, D_{s} \rightarrow \tau+N$, is $m_{D_{s}}-m_{\tau} \approx 190 \mathrm{MeV}$, and only the second summand in Eq. (3) contributes for heavier HNLs.

Let us estimate how many HNLs with $\tau$ mixing are produced as compared to those with $e$ mixing. From (3), the ratio $N_{\text {prod }}^{(\tau)} / N_{\text {prod }}^{(e)}$ is

$$
\frac{N_{\text {prod }}^{(\tau)}}{N_{\text {prod }}^{(e)}}=\frac{\sum_{D_{i}} f_{c \rightarrow D_{i}} \operatorname{Br}\left(D_{i} \rightarrow N_{\tau} X\right)+f_{c \rightarrow D_{s}} \operatorname{Br}\left(D_{s} \rightarrow \tau \bar{\nu}_{\tau}\right) \operatorname{Br}\left(\tau \rightarrow N_{\tau} X\right)}{\sum_{D_{i}} f_{c \rightarrow D_{i}} \operatorname{Br}\left(D_{i} \rightarrow N_{e} X\right)} .
$$

\footnotetext{
${ }^{1}$ To search for HNLs created in the decays of $B$ mesons at SPS, an experiment like SHiP [39] with significantly larger beam intensity delivered to the experiment and much better geometrical acceptance would be required.
} 


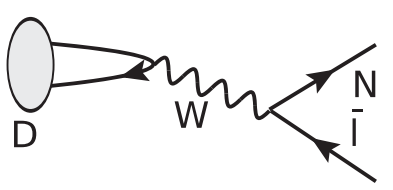

(a)

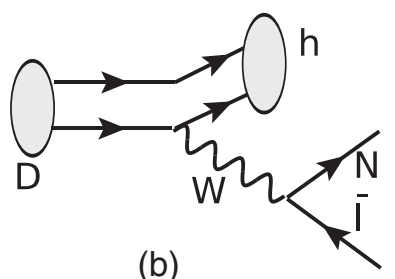

(b)

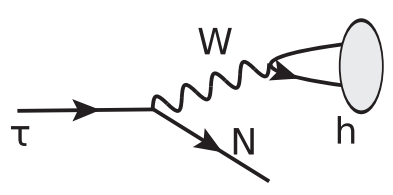

(c)

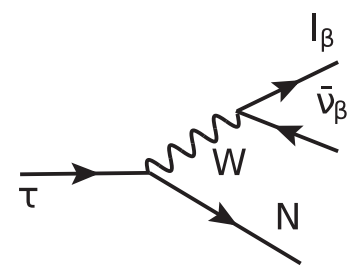

(d)

FIG. 4. Diagrams of HNL production in leptonic and semileptonic decays of $D$ mesons: $D_{s}, D^{0}, D^{ \pm}$[diagrams (a), (b)], and $\tau$ lepton, which is produced in decays of $D_{s}$ meson only [diagrams (c), (d)].

Assuming the same values of mixing angles $U_{e}^{2}=U_{\tau}^{2}$ for the two models with pure $e / \tau$ mixing, the ratio $\operatorname{Br}(\tau \rightarrow$ $\left.N_{\tau} X\right) / \sum f_{c \rightarrow D} \operatorname{Br}\left(D \rightarrow N_{e} X\right)$ varies in the 1-10 range for masses $m_{N} \lesssim 1.3 \mathrm{GeV}$ and quickly drops at the kinematic threshold $m_{N} \approx m_{\tau}$ [9]. In particular, for masses $m_{N} \gtrsim 800 \mathrm{MeV}$, where the dominant contribution to the HNL production with $e$ mixing comes from $D_{s}$, we have

$\frac{N_{\text {prod }}^{(\tau)}}{N_{\text {prod }}^{(e)}} \approx \operatorname{Br}\left(D_{s} \rightarrow \tau \bar{\nu}_{\tau}\right) \cdot \frac{\operatorname{Br}\left(\tau \rightarrow N_{\tau} X\right)}{\operatorname{Br}\left(D_{s} \rightarrow N_{e} X\right)}<4 \times 10^{-2}$.

The mass dependence of the ratio $N_{\text {prod }}^{(\tau)} / N_{\text {prod }}^{(e)}$ obtained from Eq. (4) is shown in Fig. 5.

It is important to note, that in the original analysis [19], as well as in the reanalyses [16,29], the production from $D_{s}$ has not been taken into account for the $e$ mixing. In the mass range $m_{N} \gtrsim 800 \mathrm{MeV}$, this leads to the underestimate

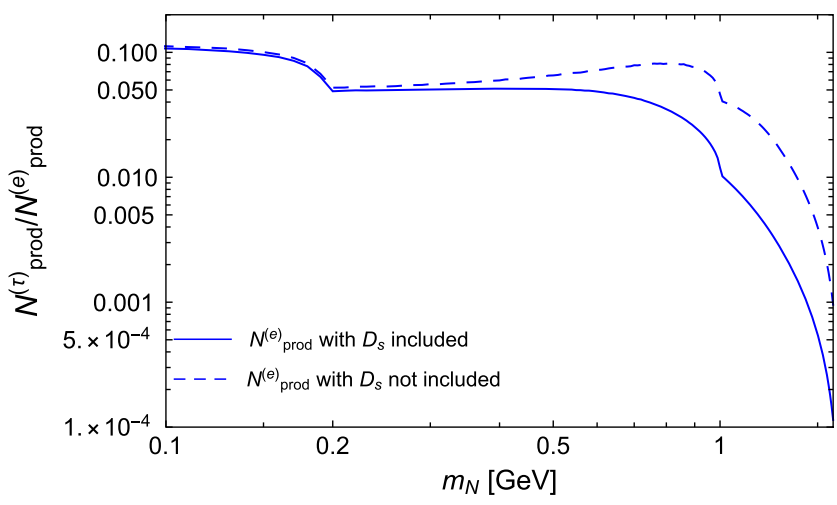

FIG. 5. The HNL mass dependence of the ratio of the numbers of produced HNLs with pure $\tau$ and $e$ mixing $N_{\text {prod }}^{(\tau)} / N_{\text {prod }}^{(e)}$; see Eq. (4), assuming the same values of the mixing angles $U_{e}^{2}=U_{\tau}^{2}$ for the two models. The solid line corresponds to $N_{\text {prod }}^{(e)}$ calculated keeping the production from all $D$ mesons $D^{+}, D^{0}, D_{s}$, while the dashed line corresponds to the estimate of $N_{\text {prod }}^{(e)} \equiv N_{\text {prod }}^{\text {CHARM }}$ calculated without the contribution of $D_{s}$, as has been done in the analysis [19] by the CHARM collaboration (see text for details). The kinks at $m_{N}=m_{D_{s}}-m_{\tau} \approx 200 \mathrm{MeV}$ an $m_{N} \approx m_{\tau}-$ $m_{\rho} \approx 1 \mathrm{GeV}$ correspond to kinematic thresholds of the production channels $D_{s} \rightarrow N+\tau, \tau \rightarrow N+\rho$, correspondingly. of the number of produced HNLs, $N_{\text {prod }}^{\mathrm{CHARM}}$, by a factor $1 / 6$ (see Fig. 4).

\section{B. Decays and their detection}

For a given number of produced HNLs, the number of detected events $N_{\text {events }}^{(\alpha)}$ for the given mixing $\alpha$ depends on

(1) Geometrical factors - in order to be detected, produced HNLs need to point in the angular coverage of the CHARM decay volume, decay inside it, and their decay products must then reach the detector and be successfully reconstructed. These factors are: geometrical acceptance $\epsilon_{\text {geom }}$, i.e., the fraction of produced HNLs traveling in the direction of the CHARM detector; the mean HNL gamma factor $\gamma_{N}$; the decay acceptance $\epsilon_{\text {decay }}$, i.e., the fraction of HNL decay products that point to the CHARM detector for HNLs that decay inside the fiducial volume.

(2) The branching ratio $\operatorname{Br}\left(N_{\alpha} \rightarrow l^{+} l^{\prime-} \nu\right)$ of the channels $N_{\alpha} \rightarrow e^{+} e^{-} \nu, N_{\alpha} \rightarrow \mu^{+} \mu^{-} \nu, N_{\alpha} \rightarrow e^{-} \mu^{+} \nu$ (and their charge conjugated counterparts) used for detection at CHARM [19].

The formula for $N_{\text {events }}^{(\alpha)}$ is:

$$
\begin{aligned}
N_{\text {events }}^{(\alpha)}= & N_{\text {prod }}^{(\alpha)} \cdot \epsilon_{\text {geom }}^{(\alpha)} \cdot \sum_{l, l^{\prime}=e, \mu} P_{\text {decay }}^{(\alpha)} \cdot \operatorname{Br}\left(N_{\alpha} \rightarrow l l^{\prime} \nu\right) \\
& \cdot \epsilon_{\text {det }, l l^{\prime}} \cdot \epsilon_{\text {decay }}^{(\alpha)},
\end{aligned}
$$

where $P_{\text {decay }}^{(\alpha)}=e^{-l_{\min } / c \tau_{N}^{(\alpha)} \gamma_{N}^{(\alpha)}}-e^{-\left(l_{\min }+l_{\text {fid }}\right) / c \tau_{N}^{(\alpha)} \gamma_{N}^{(\alpha)}}$ is the decay probability, and $\epsilon_{\mathrm{det}, l l^{\prime}}$ is the reconstruction efficiency for the given channel.

We will see below that geometrical factors are the same for $e, \mu$, and $\tau$ mixing, while the branching ratio is smaller for the $\tau$ mixing channels, as in the former case both decays via the charged and neutral currents are relevant, while in the latter only the neutral current contributes.

Let us start by considering the lower bound of the sensitivity of the CHARM experiment, i.e., the minimal mixing angles that it may probe (the upper bound will be discussed in Sec. IV). In this regime, the decay length of the HNL $c \tau_{N}^{(\alpha)} \gamma_{N}^{(\alpha)}$ is much larger than the geometric scale of the 
experiment, $\quad c \tau_{N}^{(\alpha)} \gamma_{N}^{(\alpha)} \gg l_{\text {min }}+l_{\text {fid }} \approx 515 \mathrm{~m}$. Then $P_{\text {decay }}^{(\alpha)} \approx \frac{l_{\text {fid }}}{c \gamma_{N}^{(\alpha)}} \cdot \Gamma\left(N_{\alpha}\right)$, where $\Gamma\left(N_{\alpha}\right)$ is the total decay width, and it is convenient to rewrite Eq. (6) in the form

$$
\begin{aligned}
N_{\text {events }}^{(\alpha)} \approx & N_{\text {prod }}^{(\alpha)} \times \epsilon_{\text {geom }}^{(\alpha)} \cdot \sum_{l, l^{\prime}=e, \mu} \frac{l_{\text {fid }}}{c \gamma_{N}^{(\alpha)}} \\
& \cdot \Gamma\left(N_{\alpha} \rightarrow l l^{\prime} \nu\right) \epsilon_{\mathrm{det}, l l^{\prime}} \cdot \epsilon_{\mathrm{decay}}^{(\alpha)},
\end{aligned}
$$

where $\Gamma\left(N_{\alpha} \rightarrow l^{+} l^{\prime-} \nu\right)$ is the decay width into the dilepton pair $l l^{\prime}$.

We will first discuss the difference in $\Gamma\left(N_{\alpha} \rightarrow l^{+} l^{\prime-} \nu\right)$ between the cases of $e$ and $\tau$ mixings. Decays into dileptons occur via charged and neutral current; see Fig. 1. For the NC mediated processes, the kinematic threshold $m_{N}>2 m_{e} \approx 1 \mathrm{MeV}$ is mixing independent. In contrast, for the CC mediated process for the $\tau$ mixing this threshold is $m_{N}>m_{\tau}+m_{e} \approx 1.77 \mathrm{GeV}$, and HNLs lighter than $\tau$ lepton may decay into dileptons only via NC.

Decay widths for the processes $N_{\alpha} \rightarrow l^{+} l^{\prime-} \nu$ for $m_{N} \gg$ $m_{l}+m_{l^{\prime}}$ may be given in the unified form

$$
\Gamma\left(N_{\alpha} \rightarrow l^{+} l^{\prime-} \nu\right)=c_{l l^{\prime} \nu}^{(\alpha)} \frac{G_{F}^{2} m_{N}^{5}}{192 \pi^{3}},
$$

where the coefficients $c_{l l^{\prime} \nu}^{(\alpha)}$ are given in Table I [9]. For $N_{e}$, the largest decay width is $\Gamma\left(N_{e} \rightarrow \mu^{+} e^{-} \nu_{\mu}\right)$, where only $\mathrm{CC}$ contributes. The width $\Gamma\left(N_{e} \rightarrow e^{+} e^{-} \nu_{e}\right)$ is smaller:

$$
\Gamma\left(N_{e} \rightarrow e^{-} e^{+} \nu_{e}\right) / \Gamma\left(N_{e} \rightarrow e^{-} \mu^{+} \nu_{\mu}\right) \approx 0.59,
$$

because both $\mathrm{NC}$ and $\mathrm{CC}$ contribute in this process and interfere destructively. The smallest width is $\Gamma\left(N_{e} \rightarrow \mu^{+} \mu^{-} \nu_{e}\right)$, with the process occurring only via NC. For $N_{\tau}$, there is no process $N_{\tau} \rightarrow e \mu \nu$, while in the process $N_{\tau} \rightarrow e^{+} e^{-} \nu_{\tau}$ only $\mathrm{NC}$ contributes, and thus the width is smaller than for $N_{e}$ :

$$
\Gamma\left(N_{\tau} \rightarrow e^{+} e^{-} \nu_{\tau}\right) / \Gamma\left(N_{e} \rightarrow e^{+} e^{-} \nu_{e}\right) \approx 0.22
$$

TABLE I. The values of $c_{l l^{\prime} \nu}^{(\alpha)}$ in Eq. (8) for different decay processes. For the process $N_{e} \rightarrow e^{+} e^{-} \nu_{e}$, we also provide the value obtained if including the charged current (CC) contribution only-the assumption used in [19].

\begin{tabular}{lc}
\hline \hline Process & $c_{l l^{\prime} \nu}^{(\alpha)}$ \\
\hline$N_{e / \tau} \rightarrow \mu^{+} \mu^{-} \nu_{e / \tau}$ & $\frac{1}{4}\left(1-4 \sin ^{2} \theta_{W}+8 \sin ^{4} \theta_{W}\right) \approx 0.13$ \\
$N_{\tau} \rightarrow e^{+} e^{-} \nu_{\tau}$ & $\frac{1}{4}\left(1-4 \sin ^{2} \theta_{W}+8 \sin ^{4} \theta_{W}\right) \approx 0.13$ \\
$N_{e} \rightarrow e^{-} \mu^{+} \nu_{\mu}$ & 1 \\
$N_{e} \rightarrow e^{+} e^{-} \nu_{e}$ & $\frac{1}{4}\left(1+4 \sin ^{2} \theta_{W}+8 \sin ^{4} \theta_{W}\right) \approx 0.59$ \\
$N_{e} \rightarrow e^{+} e^{-} \nu_{e}$ (CC) & 1 \\
\hline \hline
\end{tabular}

For the decay into a dimuon pair, we have $\Gamma\left(N_{\tau} \rightarrow \mu^{+} \mu^{-} \nu_{\tau}\right)=\Gamma\left(N_{e} \rightarrow \mu^{+} \mu^{-} \nu_{e}\right)$.

As a result, for $m_{N} \gg m_{\mu}$ the ratio of the factors $\sum_{l, l^{\prime}} \Gamma\left(N_{\alpha} \rightarrow l l^{\prime} \nu\right) \epsilon_{\mathrm{det}, l l^{\prime}}$ entering Eq. (7) is given by

$$
\frac{\sum_{l} \Gamma\left(N_{\tau} \rightarrow l l\right) \epsilon_{\mathrm{det}, l l}}{\sum_{l, l^{\prime}} \Gamma\left(N_{e} \rightarrow l l^{\prime}\right) \epsilon_{\mathrm{det}, l l^{\prime}}} \approx 0.16 .
$$

Here and below, we use the values of the efficiencies $\epsilon_{\mathrm{det}, l l^{\prime}}$ as reported in [19] for the HNL mass $m_{N}=1 \mathrm{GeV}$ : $\epsilon_{\mathrm{det}, e e}=0.6, \epsilon_{\mathrm{det}, e \mu}=0.65, \epsilon_{\mathrm{det}, \mu \mu}=0.75$.

In the original analysis for the $e$ mixing by the CHARM collaboration $[18,19]$, the Dirac nature of HNLs has been assumed (the decay widths are twice smaller), and only the $\mathrm{CC}$ interactions have been considered. Instead of Eq. (11), the ratio becomes

$$
\frac{2 \sum_{l} \Gamma\left(N_{\tau} \rightarrow l l\right) \epsilon_{\mathrm{det}, l l}}{\sum_{l, l^{\prime}} \Gamma_{\mathrm{CC}}\left(N_{e} \rightarrow l l^{\prime}\right) \epsilon_{\mathrm{det}, l l^{\prime}}} \approx 0.27
$$

Let us now discuss geometric factors $\epsilon_{\text {geom }}, \gamma_{N}, \epsilon_{\text {decay }}$. It turns out that they depend on the mixing pattern weakly, and as a result the geometry does not influence the relative yield of events for $e$ and $\tau$ mixing. Indeed, as was mentioned in Sec. III A, HNLs with $\tau$ mixing are produced in decays of $\tau$ leptons, that originate from decays of $D_{s}$. Since $m_{\tau} \simeq m_{D_{s}}$, the angle-energy distribution of $\tau$ leptons is the same as of $D_{s}$ (and hence also other $D$ mesons), whose decays produce HNLs with $e$ mixing. The kinematics of the HNL production from $D$ and $\tau$ are similar: twobody decays (a), (c) and three-body decays (b), (d) in Fig. 4 differ mainly because of the replacement of a neutrino or a lepton with a hadron $h=\pi, K$. However, since $m_{h} \ll m_{\tau, D}$, the replacement does not lead to the difference in the distribution of produced HNLs. In addition, heavy HNLs with masses $m_{N} \simeq 1 \mathrm{GeV}$ share the same distribution as their mother particles, and any difference disappears. Therefore, the values $\epsilon_{\text {geom }}, \gamma_{N}$ for different mixing are the same with good precision. Next, HNL decays contain the same final states independently of the mixing, and $\epsilon_{\text {decay }}$ can also be considered the same.

To summarize, the ratio $N_{\text {events }}^{(\tau)} / N_{\text {events }}^{(e)}$ is determined only by the difference in phenomenological parameters$N_{\text {prod }}^{(\alpha)}$ and $\Gamma\left(N_{\alpha} \rightarrow l l^{\prime} \nu\right):$

$$
\frac{N_{\text {events }}^{(\tau)}}{N_{\text {events }}^{(e)}} \simeq \frac{N_{\text {prod }}^{(\tau)}}{N_{\text {prod }}^{(e)}} \times \frac{\sum_{l} \Gamma\left(N_{\tau} \rightarrow l l \nu\right) \epsilon_{\text {det }, l l}}{\sum_{l, l^{\prime}} \Gamma\left(N_{e} \rightarrow l l^{\prime} \nu\right) \epsilon_{\text {det }, l l^{\prime}}}
$$

To compare with the estimate of the number of events for the $e$ mixing made by the CHARM collaboration in [19], $N_{\text {events }}^{\text {CHARM }}$, we need to take into account their assumptions on the description of HNL production and decays [see the discussion around Eqs. (4) and (12)]. The resulting ratio is 


$$
\frac{N_{\mathrm{events}}^{(\tau)}}{N_{\mathrm{events}}^{\mathrm{CHARM}}} \simeq \frac{N_{\mathrm{prod}}^{(\tau)}}{N_{\mathrm{prod}}^{\mathrm{CHARM}}} \cdot \frac{2 \sum_{l} \Gamma\left(N_{\tau} \rightarrow l l \nu\right) \epsilon_{\mathrm{det}, l l}}{\sum_{l, l^{\prime}} \Gamma_{\mathrm{CC}}\left(N_{e} \rightarrow l l^{\prime} \nu\right) \epsilon_{\mathrm{det}, l l^{\prime}}} .
$$

\section{RESULTS}

Let us now derive the CHARM sensitivity to the $\tau$ mixing. In [19], it has been shown that the dilepton decay signature at CHARM is background free. Therefore, 90\% CL sensitivity to each mixing is given by the condition

$$
N_{\text {events }}^{(e, \mu, \tau)}>2.3
$$

Let us define $U_{\text {lower,CHARM }}^{2}$ as the smallest mixing angle for which the condition (15) is satisfied for the assumptions of the original analysis of [19] [see the discussion above Eq. (14)]. As the number of detected events at the lower bound $N_{\text {events }}^{(\alpha)}$ scales with the mixing angle as $N_{\text {events }}^{(\alpha)} \propto U_{\alpha}^{4}$ (where $U_{\alpha}^{2}$ comes from the production and another $U_{\alpha}^{2}$ from decay probability), we can use Eqs. (14) and (4) to obtain the lower bound of the sensitivity to the $\tau$ mixing, $U_{\tau, \text { lower }}^{2}$, by rescaling the results reported in [19]:

$$
\left.\frac{U_{\tau, \text { lower }}^{4}}{U_{\text {lower }}^{4 \text { CHARM }}} \simeq \frac{N_{\text {prod }}^{\text {CHARM }}}{N_{\text {prod }}^{(\tau)}} \cdot \frac{\sum_{l, l^{\prime}} \Gamma_{\mathrm{CC}}\left(N_{e} \rightarrow l l^{\prime} \nu\right) \epsilon_{\mathrm{det}, l l^{\prime}}}{\sum_{l} \Gamma\left(N_{\tau} \rightarrow l \bar{l} \nu\right) \epsilon_{\mathrm{det}, l l}}\right|_{U_{e}=U_{\tau}} .
$$

Using the ratio $N_{\text {prod }}^{\text {CHARM }} / N_{\text {prod }}^{(\tau)}$ from Eq. (4) (see also Fig. 5), and the ratio of decay widths from Eq. (12), we conclude that in the mass range $m_{N}>200 \mathrm{MeV}$ the lower bound for the $\tau$ mixing is a factor 10-100 weaker than the lower bound for the $e$ mixing reported in [19]. In the domain $m_{D_{s}}-m_{\tau}<m_{N}<290 \mathrm{MeV}$, we validate the rescaled bound (16) by comparing it with the CHARM sensitivity to the $\tau$ mixing from [20]; see Appendix A.

At the upper bound of the sensitivity, the dependence of the number of events on $U_{\alpha}^{2}$ is complicated and the sensitivity cannot be obtained by rescaling the results of [19]. Therefore, we independently compute the number of decay events at CHARM for HNLs with $e$ and $\tau$ mixing and then calculate the sensitivity numerically using Eq. (15); see Appendix A. In order to validate this estimate, we compare the resulting sensitivity for the $\tau$ mixing with the rescaled bound (16), and find that they are in very good agreement (Fig. 7). Also, we compare our estimate for the $e$ mixing with the CHARM sensitivity to the $e$ mixing from [19]. In our estimates, we include neutral current interactions, the production from $D_{s}$ mesons, and assume that HNLs are Majorana particles. Due to these reasons, we find that for small mixing angles $U_{e}$ and above $m_{N} \gtrsim 1 \mathrm{GeV}$, the bound imposed by CHARM may be actually improved by up to a factor 3-4. Let us comment on errors of our estimates. We used the values of reconstruction efficiencies $\epsilon_{\text {rec, } l l}$ reported in [19] for the HNL mass $m_{N}=1 \mathrm{GeV}$. Hence, the calculation may be further refined by including HNL mass dependent reconstruction efficiencies. However, as the study [20] performed for the $\tau$ mixing and masses $m_{N}<290 \mathrm{MeV}$ has shown similar efficiency, we do not expect any significant changes.

Our final results for the $\tau$ mixing are given in Fig. 6, where we show the domain excluded by previous experiments together with updated CHARM bounds, and the sensitivity of the future experiments mentioned in Sec. I, together with SHiP [40]. Comparing with Fig. 2, we find that in the mass range $290 \mathrm{MeV}<m_{N}<1.6 \mathrm{GeV}$ our results improve previously reported bounds on the mixing angle $U_{\tau}^{2}$ by two orders of magnitude. In particular, it excludes a large part of the parameter space that was suggested to be probed by the future experiments. For instance, Belle II, FASER, DarkQuest, and the double bang signature at IceCube have sensitivity only in the narrow domain above the CHARM upper bound, while NA62 may slightly push probed angles to lower values. The same is the case for the sensitivity of KM3NeT in the regime of the
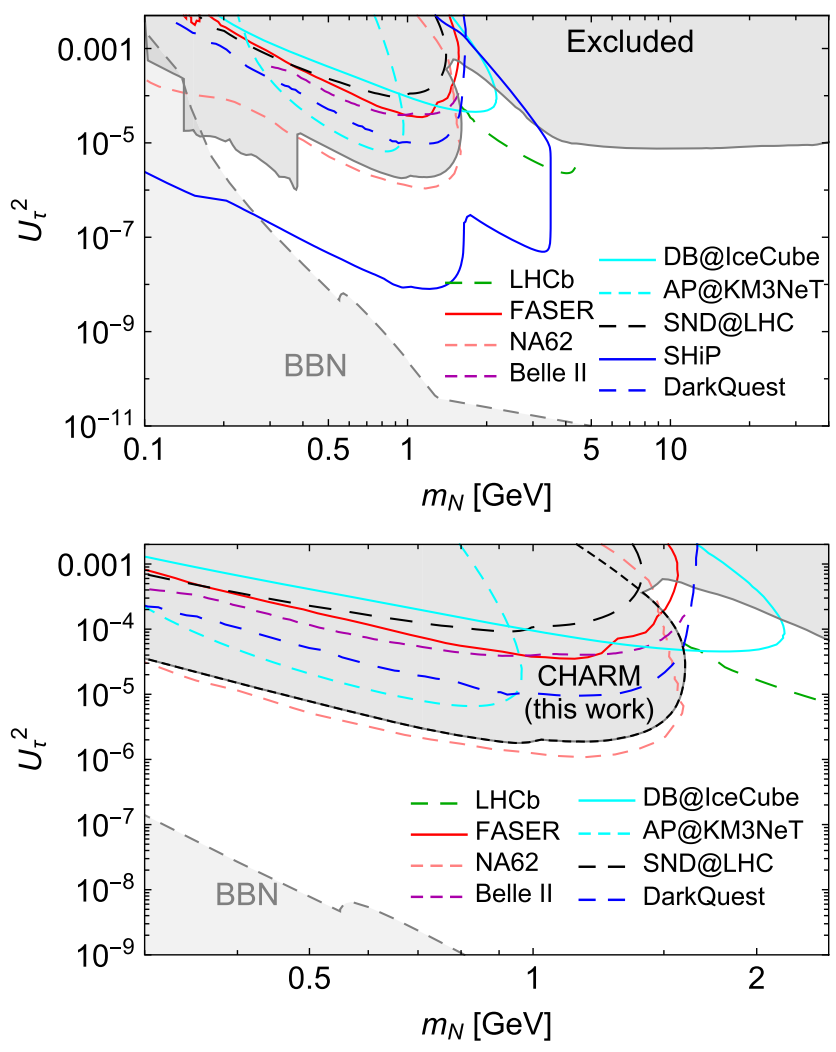

FIG. 6. Parameter space of a single Majorana HNL that mixes with $\nu_{\tau}$. The excluded region is a combined reach of the DELPHI [25] and CHARM experiments (our result), which is also highlighted by the short dashed black line. Bounds from BBN are reproduced from $[12,14]$. The sensitivity of future experiments is also shown (see text around Fig. 2 for details). The top panel covers the HNL mass region $m_{N}=0.1-35 \mathrm{GeV}$, while the bottom panel is a zoom in of the mass domain $m_{N}=\mathcal{O}(1 \mathrm{GeV})$. 
atmospheric beam dump, which we estimate in Appendix B. Significant progress in testing the mixing of HNLs with $\nu_{\tau}$ can be achieved by LHCb, which probes the complementary mass range $m_{N}>2 \mathrm{GeV}$, and dedicated intensity frontier experiments, with SHiP being optimal for searches of HNLs from decays of $D$ mesons and $\tau$ leptons.

\section{ACKNOWLEDGMENTS}

We thank Y. Cheipesh for careful reading and improving the quality of the manuscript. This project has received funding from the European Research Council (ERC) under the European Union's Horizon 2020 research and innovation programme (GA 694896) and from the NWO Physics Vrij Programme "The Hidden Universe of Weakly Interacting Particles", No. 680.92.18.03, which is partly financed by the Dutch Research Council NWO.

\section{APPENDIX A: CHARM SENSITIVITY BASED ON NUMBER OF DECAY EVENTS ESTIMATE}

The number of decay events for the pure $\alpha$ mixing at CHARM is given by the formula

$N_{\text {events }}^{(\alpha)}=\sum_{X=D, \tau} N_{X} \cdot \operatorname{Br}\left(X \rightarrow N_{\alpha}\right) \int d E d \theta d z \cdot f_{N_{\alpha}}^{X}(E, \theta) \frac{e^{-l(z) / c \tau_{N} \gamma}}{c \tau_{N}^{(\alpha)} \gamma} \frac{\Delta \phi(\theta, z)}{2 \pi} \cdot \epsilon_{\text {decay }}(\theta, z, E) \cdot \operatorname{Br}\left(N_{\alpha} \rightarrow l \bar{l}^{\prime}\right) \cdot \epsilon_{\text {det }, l l^{\prime}}$

Here,

$N_{D_{i}}=N_{\mathrm{PoT}} \times \chi_{c \bar{c}} \times f_{c \rightarrow D_{i}}, \quad N_{\tau}=N_{D_{s}} \times \operatorname{Br}\left(D_{s} \rightarrow \tau \bar{\nu}_{\tau}\right)$

are the total numbers of $D$ mesons $\left(D_{i}=D_{s}, D^{+}, D^{0}\right)$ and $\tau$ leptons, with $N_{\text {PoT }}=2.4 \times 10^{18}$ being the total number of proton-target collisions at CHARM and $\chi_{c \bar{c}} \approx 4 \times 10^{-3}$ the production fraction of the $c \bar{c}$ at SPS energies for a thick target [41]. $\mathrm{Br}_{D_{s} \rightarrow \tau} \approx 5.43 \%$ [42] and $f_{c \rightarrow D_{i}}$ are given from [40]. $f_{N_{\alpha}}^{X}$ is the distribution of HNLs produced in decays of $X$ particles in polar angle and energy. $z \in$ $(480,515) \mathrm{m}$ is the longitudinal distance, $\theta \in$ $(3.5 / 515,6.5 / 515)$ is the polar angle coverage of the end of the CHARM's decay volume, while $\Delta \phi(\theta) / 2 \pi$ is the azimuthal acceptance for HNLs decaying inside the decay volume. $\epsilon_{\text {decay }}$ is the decay acceptance-a fraction of decay products of HNLs that both point to the detector. Finally, $\epsilon_{\mathrm{det}, l l^{\prime}}$ are reconstruction efficiencies for leptonic decays: $\epsilon_{e e} \approx 60 \%, \epsilon_{\mu \mu} \approx 75 \%$, and $\epsilon_{e \mu} \approx 65 \%$, which we use from [19].

Computing of $f_{N_{\alpha}}(E, \theta)$ requires knowing the distribution of $D$ mesons and $\tau$ leptons $f_{\tau}(E, \theta)$ produced at the CHARM target. We approximate $f_{\tau}$ by the distribution of $D_{s}$ mesons, while for the distribution of $D$ mesons we use FairShip simulations [41] for collisions of the SPS proton beam with a thick Tungsten target. ${ }^{2}$
The distribution of HNLs $f_{N_{\alpha}}^{X}(E, \theta)$ has been obtained from $f_{X}(E, \theta)$ semianalytically using the method from [43].

We have estimated $\epsilon_{\text {decay }}$ by using a toy simulation for decays of HNLs inside the decay volume into three massless particles, and requiring the momenta of the two charged leptons to point towards the end of the decay volume. The acceptances are shown in Fig. 7.

In order to obtain the excluded domain, we assume the absence of background and require $N_{\text {events }}>2.3$, which corresponds to the $90 \%$ C.L.

The comparison of this estimate with the rescale from Sec. IV and [20] is shown in Fig. 7. We find that the estimates are in very good agreement. We also show our estimate of the CHARM bounds on the $e$ mixing, which differs from the bounds obtained from [19] by including the production from $D_{s}$ mesons, which dominates masses $m_{N} \gtrsim 700 \mathrm{MeV}$ (see also Fig. 5). The resulting sensitivity at the lower bound improves by up to a factor 3-4 for this mass region.

\section{APPENDIX B: SENSITIVITY OF KM3NeT TO HNLs}

In this Appendix, we demonstrate the potential of the neutrino telescope $\mathrm{KM} 3 \mathrm{NeT}$ [36] to probe the parameter space of HNLs.

HNLs may be numerously produced in decays of $\tau$ leptons, originated from the collisions of high-energy cosmic protons with the well-known spectrum

$$
\frac{d \Phi}{d \Omega d t d S d E_{p}} \approx \begin{cases}1.7 E_{p, \mathrm{GeV}}^{-2.7} \mathrm{GeV}^{-1} \mathrm{sr}^{-1} \mathrm{~cm}^{-2} \mathrm{~s}^{-1}, & E_{p}<5 \times 10^{6} \mathrm{GeV} \\ 174 E_{p, \mathrm{GeV}}^{-3} \mathrm{GeV}^{-1} \mathrm{sr}^{-1} \mathrm{~cm}^{-2} \mathrm{~s}^{-1}, & E_{p} \geq 5 \times 10^{6} \mathrm{GeV}\end{cases}
$$

\footnotetext{
${ }^{2}$ Although at CHARM the target material is different, we believe that it is still a reasonable approximation.
} 

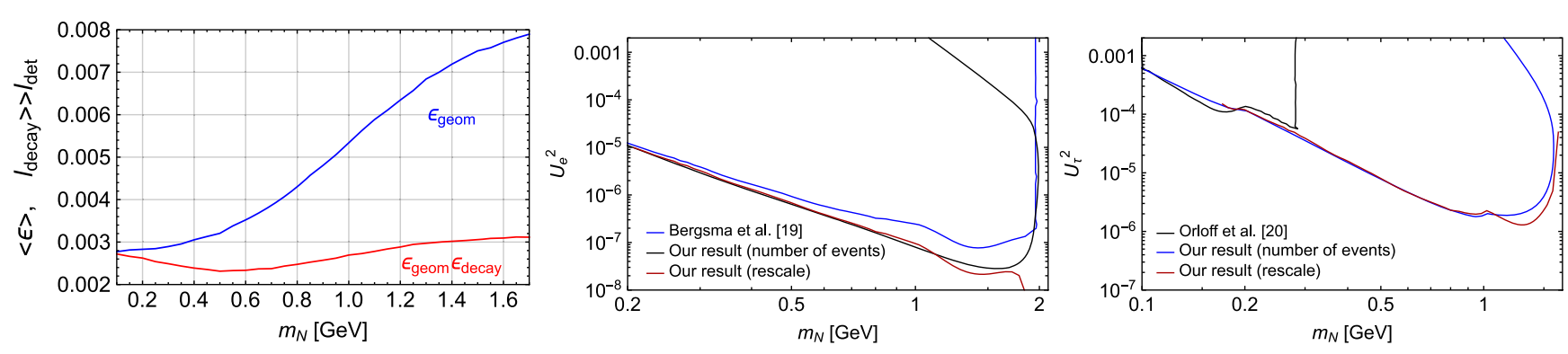

FIG. 7. The left panel: fraction of HNLs that point towards the detector (blue line) and fraction of HNLs whose decay products point towards the detector (red line). The middle and right panels: comparison of our estimates of the constraint from the CHARM experiment on the pure $e$ (the middle panel) and $\tau$ mixing (the right panel), with bounds reported in [19,20]. We show two estimates: the red line corresponds to the rescale of the bound on the $e$ mixing from [19] (see Sec. IV for details), while the blue line is our independent estimate based on Eq. (A1).

with atmospheric particles. If having significantly large lifetimes, produced HNLs may enter the detector volume of KM3NeT located deep in the Mediterranean Sea and decay there.

In order to probe the parameter space of HNLs, it is necessary to distinguish their decays from interactions of SM particles also produced in the atmosphere: neutrinos and muons. KM3NeT may only distinguish two event types: tracklike, which corresponds to muons penetrating through the detector volume, and cascadelike, which originates from other particles such as electrons and hadrons. Scatterings of neutrinos inside the detector volume produce cascadelike (if no high-energy muons are produced) or combined cascadelike + tracklike signature (if high-energy muons are produced), while penetrating atmospheric muons give rise to tracklike signature. A possible way to distinguish these events from HNLs is to look for their decays into a dimuon pair, $N \rightarrow \mu \bar{\mu} \nu_{\tau}$. They produce a signature of two tracks originated from one point inside the detector volume. Detectors of KM3NeT have energy and angular resolution sufficient precise for resolving the two tracks down to energies of $\simeq 10 \mathrm{GeV}$ [44]. Therefore, we believe that the dimuon signature may be reconstructed in the background free regime with high efficiency.

\section{Analytic estimates: Comparison with CHARM}

Now, let us discuss the sensitivity of KM3NeT to HNLs. Let us first compare the amount of HNL decay events at CHARM and KM3NeT for given value of the mixing angle at the lower bound of the sensitivity using simple analytic estimates. According to Eq. (7), for the ratio of decay events at these experiments we have

$$
\begin{aligned}
& \frac{N_{\text {events,CHARM }}^{(\tau)}}{N_{\text {events,KM3NeT }}^{(\tau)}} \simeq \frac{N_{c \bar{c}}^{\mathrm{CHARM}} \cdot \epsilon_{\mathrm{geom}}^{\mathrm{CHARM}} \cdot \epsilon_{\mathrm{decay}}^{\mathrm{CHARM}}}{N_{c \bar{c}}^{\mathrm{KMNeT}}} \times \frac{l_{\mathrm{fid}}^{\mathrm{CHARM}}}{l_{\text {fid }}^{\mathrm{KM} 3 \mathrm{NeT} T}} \\
& \times \frac{\gamma_{N}^{\mathrm{KM} 3 \mathrm{NeT}}}{\gamma_{N}^{\mathrm{CHARM}}} \times \frac{\sum_{l=e, \mu} \Gamma\left(N_{\tau} \rightarrow l l\right) \epsilon_{\mathrm{det}, l l}}{\Gamma\left(N_{\tau} \rightarrow \mu \mu\right)} .
\end{aligned}
$$

Here, $N_{c \bar{c}}^{\mathrm{CHARM}} \cdot \epsilon_{\text {geom }}^{\mathrm{CHARM}} \cdot \epsilon_{\text {decay }}^{\mathrm{CHARM}} \simeq 2 \times 10^{13}$ (see, Fig. 7 is the number of $c \bar{c}$ pairs detectable fraction of HNL decay events at CHARM). $N_{c \bar{c}}^{\mathrm{KM} 3 \mathrm{NeT}}$ is the amount of $c \bar{c}$ pairs produced in the upper hemisphere propagating to KM3NeT,

$$
\begin{array}{rl}
N_{c \bar{c}}^{\mathrm{KM} 3 \mathrm{NeT}} \simeq 2 & 2 \pi \times 1 \mathrm{~km}^{2} \times 5 \text { years } \\
\times \int \frac{d \Phi}{d \Omega d t d S d E_{p}} \cdot \frac{\sigma_{p p \rightarrow c \bar{c} X}}{\sigma_{p p, \text { total }}} d E_{p} \simeq 10^{12},
\end{array}
$$

where $\sigma_{p p \rightarrow c \bar{c} X}\left(E_{p}\right)$ is the energy-dependent charm production cross section which we use from FONLL [45] and from [46], and $\sigma_{p p \text {,total }}$ is the total pp-cross-section, which we use from [47]. The integrand in (B3) is the product of two competing factors: $\frac{d \Phi}{d \Omega d t d S d E_{p}}$, which decreases with the proton's energy, and $\sigma_{p p \rightarrow c \bar{c} X}\left(E_{p}\right)$, which increases; see Fig. 8.

We approximate the ratio of the mean HNL $\gamma$ factors by the ratio of the mean $\gamma$ factors of $D$ mesons:

$$
\gamma_{N}^{\mathrm{KM} 3 \mathrm{NeT}} / \gamma_{D_{s}}^{\mathrm{CHARM}} \simeq \gamma_{D_{s}}^{\mathrm{KM} 3 \mathrm{NeT}} / \gamma_{D_{s}}^{\mathrm{CHARM}} \simeq 3,
$$

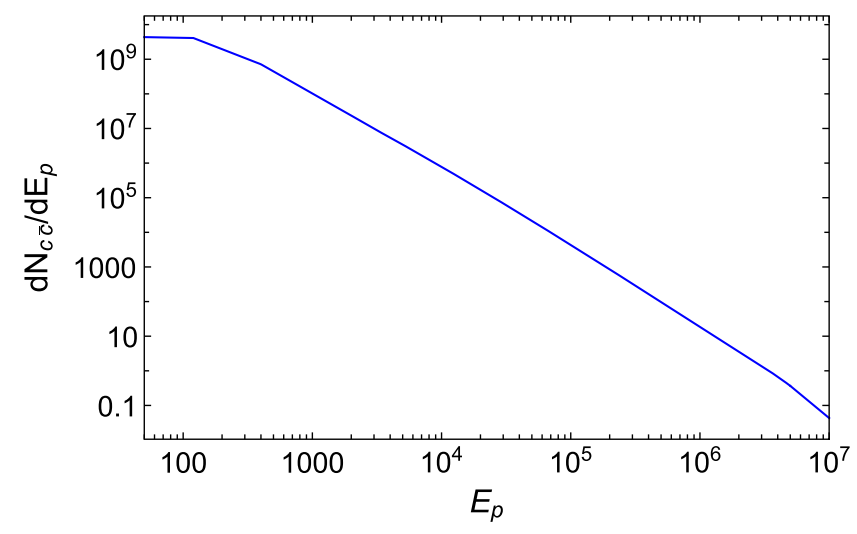

FIG. 8. The integrand of Eq. (B3). 
where we calculate $\gamma_{D_{s}}^{\mathrm{KM} 3 \mathrm{NeT}}$ using the $c \bar{c}$ distribution $\frac{d \Phi}{d \Omega d t d S d E_{p}} \cdot \sigma_{p p \rightarrow c \bar{c} X}$, assuming that $E_{D} \approx E_{p} / 2$.

Using the fiducial lengths $l_{\text {fid }}^{\text {CHARM }}=35 \mathrm{~m}$ and $l_{\text {fid }}^{\mathrm{KM} 3 \mathrm{NeT}} \simeq 1 \mathrm{~km}$, and taking into account that the last factor in Eq. (B2) is $\mathcal{O}(1)$ for $m_{N} \gg 2 m_{\mu}$, we finally obtain

$$
\frac{N_{\text {events,CHARM }}^{(\tau)}}{N_{\text {events,KM3NeT }}^{(\tau)}} \simeq 2 .
$$

Therefore, naively, even in the most optimistic case (assuming unit efficiency) the number of events are just comparable. We need more accurate estimate taking into account nonisotropic distribution of the produced HNLs.

\section{Accurate estimate}

We compute the production of $D_{s}$ mesons (and hence $\tau$ leptons) using the approach from [48]. The production was found to be maximal at $O(10 \mathrm{~km})$ height from the Earth's surface. The resulting spectrum $\frac{d \Phi_{D_{s}}}{d S d t d l d \cos (\theta) d E_{D_{s}}}$ of $D_{s}$ mesons is in good agreement with Fig. 2 from [49]. The total number of $D_{s}$ mesons produced in the direction of $\mathrm{KM} 3 \mathrm{NeT}$ during the operating time 5 years was found to be $N_{D_{s}} \simeq 5 \times 10^{10}$.

Next, we use the approach from [49] in order to estimate the sensitivity of KM3NeT. The number of decay events is

$$
N_{\text {events }} \approx S_{\mathrm{Km} 3 \mathrm{NeT}} \times T \times \int \frac{d \Phi_{D_{s}}}{d S d t d l d \cos (\theta) d E_{D_{s}}} \cdot \operatorname{Br}\left(D_{s} \rightarrow \tau \bar{\nu}_{\tau}\right) \cdot \operatorname{Br}\left(\tau \rightarrow N_{\tau} X\right) \cdot P_{\text {decay }}\left(l, E_{N}\right) d \cos (\theta) d l d E_{N},
$$

where $T=5$ years is the operating time, $S_{\mathrm{KM} 3 \mathrm{NeT}}=1 \mathrm{~km}^{2}$ is the transverse area of $\mathrm{KM} 3 \mathrm{NeT}$. The decay probability is

$$
P_{\text {decay }} \approx e^{-\left(l+l_{1}\right) / l_{\text {decay }}}-e^{-\left(l+l_{2}\right) / l_{\text {decay }}},
$$

where $l$ is the distance from the HNL production point in atmosphere, $l_{1} \approx 3 \mathrm{~km}$ is the distance from the surface of Earth to the KM3NeT detector, while $l_{2}=l_{1}+1 \mathrm{~km}$ is the distance to the end of the KM3NeT. For simplicity, in $l_{\text {decay }}$ we set $E_{N} \approx E_{D_{s}} / 2$. In order to show the maximal reach of KM3NeT, we optimistically assume unit efficiency of the dimuon event reconstruction, and require $N_{\text {events }}>3$ during the operating period.

The resulting sensitivity shown in Fig. 6 is worse than predicted by the simple estimate by a factor of few. The reason is that at masses $m_{N} \lesssim 500 \mathrm{MeV}$ there is an additional suppression from $\operatorname{Br}(N \rightarrow \mu \mu)$, while at higher masses the scaling (7) is not valid because the lower bound is close to the upper bound.
[1] P. Minkowski, $\mu \rightarrow e \gamma$ at a rate of one out of $10^{9}$ muon decays? Phys. Lett. 67B, 421 (1977).

[2] T. Asaka, S. Blanchet, and M. Shaposhnikov, The nuMSM, dark matter and neutrino masses, Phys. Lett. B 631, 151 (2005).

[3] A. Boyarsky, M. Drewes, T. Lasserre, S. Mertens, and O. Ruchayskiy, Sterile neutrino dark matter, Prog. Part. Nucl. Phys. 104, 1 (2019).

[4] A. Boyarsky, O. Ruchayskiy, and M. Shaposhnikov, The role of sterile neutrinos in cosmology and astrophysics, Annu. Rev. Nucl. Part. Sci. 59, 191 (2009).

[5] T. Asaka and M. Shaposhnikov, The nuMSM, dark matter and baryon asymmetry of the universe, Phys. Lett. B 620, 17 (2005).

[6] T. Asaka and H. Ishida, Flavour mixing of neutrinos and baryon asymmetry of the Universe, Phys. Lett. B 692, 105 (2010).

[7] T. Asaka, S. Eijima, and H. Ishida, Kinetic equations for baryogenesis via sterile neutrino oscillation, J. Cosmol. Astropart. Phys. 02 (2012) 021.
[8] M. Shaposhnikov, Baryogenesis, J. Phys. Conf. Ser. 171, 012005 (2009).

[9] K. Bondarenko, A. Boyarsky, D. Gorbunov, and O. Ruchayskiy, Phenomenology of GeV-scale heavy neutral leptons, J. High Energy Phys. 11 (2018) 032.

[10] J. Beacham et al., Physics beyond colliders at CERN: Beyond the standard model working group report, J. Phys. G 47, 010501 (2020).

[11] P. Agrawal et al., Feebly-interacting particles: FIPs 2020 workshop report, arXiv:2102.12143.

[12] A. Boyarsky, M. Ovchynnikov, O. Ruchayskiy, and V. Syvolap, Improved BBN constraints on heavy neutral leptons, Phys. Rev. D 104, 023517 (2021).

[13] A. Boyarsky, M. Ovchynnikov, N. Sabti, and V. Syvolap, When FIMPs decay into neutrinos: The $N_{\text {eff }}$ story, Phys. Rev. D 104, 035006 (2021).

[14] N. Sabti, A. Magalich, and A. Filimonova, An extended analysis of heavy neutral leptons during big bang nucleosynthesis, J. Cosmol. Astropart. Phys. 11 (2020) 056. 
[15] K. Bondarenko, A. Boyarsky, J. Klaric, O. Mikulenko, O. Ruchayskiy, V. Syvolap et al., An allowed window for heavy neutral leptons below the kaon mass, J. High Energy Phys. 07 (2021) 193.

[16] M. Chrzaszcz, M. Drewes, T. E. Gonzalo, J. Harz, S. Krishnamurthy, and C. Weniger, A frequentist analysis of three right-handed neutrinos with GAMBIT, Eur. Phys. J. C 80, 569 (2020).

[17] M. Drewes, J. Hajer, J. Klaric, and G. Lanfranchi, NA62 sensitivity to heavy neutral leptons in the low scale seesaw model, J. High Energy Phys. 07 (2018) 105.

[18] F. Bergsma et al. (CHARM Collaboration), A search for decays of heavy neutrinos, Phys. Lett. 128B, 361 (1983).

[19] F. Bergsma et al. (CHARM Collaboration), A search for decays of heavy neutrinos in the mass range $0.5-\mathrm{GeV}$ to 2.8-GeV, Phys. Lett. 166B, 473 (1986).

[20] J. Orloff, A. N. Rozanov, and C. Santoni, Limits on the mixing of tau neutrino to heavy neutrinos, Phys. Lett. B 550, 8 (2002).

[21] E. Cortina Gil et al. (NA62 Collaboration), Search for heavy neutral lepton production in $K^{+}$decays to positrons, Phys. Lett. B 807, 135599 (2020).

[22] K. Abe et al. (T2K Collaboration), Search for heavy neutrinos with the T2K near detector ND280, Phys. Rev. D 100, 052006 (2019).

[23] D. Liventsev et al. (Belle Collaboration), Search for heavy neutrinos at Belle, Phys. Rev. D 87, 071102 (2013).

[24] C. O. Dib, J. C. Helo, M. Nayak, N. A. Neill, A. Soffer, and J. Zamora-Saa, Searching for a sterile neutrino that mixes predominantly with $\nu_{\tau}$ at $B$ factories, Phys. Rev. D 101, 093003 (2020).

[25] P. Abreu et al. (DELPHI Collaboration), Search for neutral heavy leptons produced in Z decays, Z. Phys. C 74, 57 (1997).

[26] P. Astier et al. (NOMAD Collaboration), Search for heavy neutrinos mixing with tau neutrinos, Phys. Lett. B 506, 27 (2001).

[27] R. Acciarri et al. (ArgoNeuT Collaboration), New Constraints on Tau-Coupled Heavy Neutral Leptons with Masses $m N=280-970$ MeV, Phys. Rev. Lett. 127, 121801 (2021).

[28] P. Coloma, P. A. N. Machado, I. Martinez-Soler, and I. M. Shoemaker, Double-Cascade Events from New Physics in Icecube, Phys. Rev. Lett. 119, 201804 (2017).

[29] O. Ruchayskiy and A. Ivashko, Experimental bounds on sterile neutrino mixing angles, J. High Energy Phys. 06 (2012) 100.

[30] A. Ariga et al. (FASER Collaboration), FASER's physics reach for long-lived particles, Phys. Rev. D 99, 095011 (2019).

[31] A. Boyarsky, O. Mikulenko, M. Ovchynnikov, and L. Shchutska, Searches for new physics at SND@LHC, arXiv:2104.09688.

[32] B. Batell, J. A. Evans, S. Gori, and M. Rai, Dark scalars and heavy neutral leptons at darkquest, J. High Energy Phys. 05 (2021) 049.
[33] G. Cvetič and C.S. Kim, Sensitivity bounds on heavy neutrino mixing $\left|U_{\mu N}\right|^{2}$ and $\left|U_{\tau N}\right|^{2}$ from LHCb upgrade, Phys. Rev. D 100, 015014 (2019).

[34] E. J. Chun, A. Das, S. Mandal, M. Mitra, and N. Sinha, Sensitivity of lepton number violating meson decays in different experiments, Phys. Rev. D 100, 095022 (2019).

[35] M. Atkinson, P. Coloma, I. Martinez-Soler, N. Rocco, and I. M. Shoemaker, Heavy neutrino searches through doublebang events at super-kamiokande, DUNE, and hyperkamiokande, arXiv:2105.09357.

[36] S. Adrian-Martinez et al. (KM3Net Collaboration), Letter of intent for KM3NeT 2.0, J. Phys. G 43, 084001 (2016).

[37] A. N. Diddens et al. (CERN-Hamburg-Amsterdam-RomeMoscow Collaboration), A detector for neutral current interactions of high-energy neutrinos, Nucl. Instrum. Methods 178, 27 (1980).

[38] A. Diddens, M. Jonker, J. Panman, F. Udo, J. Allaby, U. Amaldi et al., Performance of a large system of proportional drift tubes for a fine-grain calorimeter, Nucl. Instrum. Methods 176, 189 (1980).

[39] M. Anelli et al. (SHiP Collaboration), A facility to search for hidden particles (SHiP) at the CERN SPS, arXiv:1504.04956.

[40] C. Ahdida et al. (SHiP Collaboration), Sensitivity of the SHiP experiment to heavy neutral leptons, J. High Energy Phys. 04 (2019) 077.

[41] H. Dijkstra and T. Ruf (SHiP Collaboration), Heavy Flavour Cascade Production in a Beam Dump, CERN Report No. CERN-SHiP-NOTE-2015-009, 2015, https://cds.cern .ch/record/2115534.

[42] M. Tanabashi et al. (Particle Data Group Collaboration), Review of particle physics, Phys. Rev. D 98, 030001 (2018).

[43] I. Boiarska, K. Bondarenko, A. Boyarsky, M. Ovchynnikov, O. Ruchayskiy, and A. Sokolenko, Light scalar production from Higgs bosons and FASER 2, J. High Energy Phys. 05 (2020) 049.

[44] S. Adrian-Martinez et al. (KM3Net Collaboration), Letter of intent for KM3NeT 2.0, J. Phys. G 43, 084001 (2016).

[45] M. Cacciari, M. L. Mangano, and P. Nason, Gluon PDF constraints from the ratio of forward heavy-quark production at the LHC at $\sqrt{S}=7$ and $13 \mathrm{TeV}$, Eur. Phys. J. C 75, 610 (2015).

[46] F. Bezrukov and D. Gorbunov, Light inflaton Hunter's Guide, J. High Energy Phys. 05 (2010) 010.

[47] S. Alekhin et al., A facility to search for hidden particles at the CERN SPS: The SHiP physics case, Rep. Prog. Phys. 79, 124201 (2016).

[48] P. Gondolo, G. Ingelman, and M. Thunman, Charm production and high-energy atmospheric muon and neutrino fluxes, Astropart. Phys. 5, 309 (1996).

[49] C. Argüelles, P. Coloma, P. Hernández, and V. Muñoz, Searches for atmospheric long-lived particles, J. High Energy Phys. 02 (2020) 190. 